\title{
Classical Collisional Trajectories as the Source of Strong Field Double Ionization of Helium in the Knee Regime
}

\author{
Li-Bin $\mathrm{Fu}^{1}$, Jie Liu ${ }^{1,2}$, Jing Chen ${ }^{2,3}$ and Shi-Gang Chen ${ }^{1}$ \\ ${ }^{1}$ Institute of Applied Physics and Computational Mathematics, \\ P.O. Box 8009 (26), 100088 Beijing, China \\ ${ }^{2}$ CCAST (World Laboratory), P.O.Box 8730, Beijing \\ ${ }^{3}$ Institute of Theoretical Physics, Chinese Academy of Science \\ P.O. Box 2735, Beijing 100080, China
}

\begin{abstract}
In this paper, a quasistatic model is extended to describe the double ionization of Helium in intense linearly polarized field, yielding achieve an insight to the two-electron correlation effect in the ionization dynamics. Our numerical calculations reproduce the excessive double ionization and the photoelectron spectra observed experimentally both quantitatively and qualitatively. Moreover, it is shown that the classical collisional trajectories are the main source of the double ionization in the knee regime and responsible for the unusual angular distribution of the photoelectrons.
\end{abstract}

PACS numbers: $32.80 . \mathrm{Rm}, 42.50 . \mathrm{Hz}, 31.15 .-\mathrm{p}$

Recently the excessive double ionization observed in Helium experiments by Fittinghoff et al. [1], Walker et al. [2], and Sheehy et al. [3] draws much attention to the multiple-electron dynamics in the laser-atom interaction. In these experiments the single ionization yields of He in a linearly polarized field is accurately predicted by the single active electron (SAE) approximation [2], well described by the Ammosov-Delone-Krainov (ADK) tunneling theory [4]. However, the case of double ionization is more complicated. In the regime of very high intensities $\left(I>10^{16} \mathrm{~W} / \mathrm{cm}^{2}\right)$ where strong double ionization occurs, the double ionization keeps in good agreement with the sequential SAE models as that in the lower intensities regime $\left(I<10^{14} \mathrm{~W} / \mathrm{cm}^{2}\right)$. The double ionization yield deviates seriously from the sequential SAE model and shows a great enhancement in a "knee" regime $\left[(0.8-3.0) \times 10^{15} \mathrm{~W} / \mathrm{cm}^{2}\right]$, where the $\mathrm{He}^{2+} / \mathrm{He}^{+}$yields ratio is close to a constant: 0.002 . This surprising large yields of the double ionization obviously indicates that the sequential ionization is no longer the dominating process in this regime and the electron-electron correlation has to be taken into account.

Both the "shake-off" model and the "recollision" model are suggested to describe the electron's correlation [1, 3,5, 6]. However, none of the two nonsequential ionization (NSI) mechanisms can completely explain the experimental observations. For the "shake-off" model, it can not give the reason for the decrease in the double ionization yields as the polarization of the laser field departs from linear [7 9 . In the "recollision" model, the returning electrons are known to have a maximum classical kinetic energy of $\sim 3.2 U_{p}\left(U_{p}=e^{2} F^{2} / 4 m_{e} \omega^{2}\right)$, so one can determine a minimum intensity required for the rescattering electron to have enough energy to excite the inner electron. But the double ionization yields observed in experiments have no such an intensity threshold. In fact, the double ionization process is rather complicated and subtle, both of the two NSI processes and the sequential ionization contribute to the double ionization yields and may dominate in the different regimes.

The experiments on the double ionization of Helium are mainly confined in the tunneling regime, i.e. the ratio between the tunneling time of the outer electron and the inverse optical frequency (Keldysh parameter) is less than 1. In this regime, the quasistatic model [5] provides a perfect description for the Hydrogen-like atoms in the intense fields and successfully explain the most nonlinear phenomena observed experimentally [5, 10,11]. Inspired by this success, in this paper we extend to develop a 3D quasistatic model (a two step process) to investigate the mechanism of the double ionization of Helium by tracing the classical trajectories of the two correlated electrons. We attribute the double ionization to the classical collisional trajectories: Distinct trajectory configurations corresponding to the "shake-off" and "recollision" mechanism contribute to the nonsequential double ionization of helium. Our numerical simulations successfully reproduce the excessive double ionization and the photoelectron spectra observed experimentally in the knee regime. The intuitionistic pictures of the double ionization will be provided by this model.

As a beginning, we present the improved two-step quasistatic model adopted in our calculations. The first step that the outer electron tunnels free, is treated by the tunneling ionization theory generalized by Delone et al. [12]. In the second step, the evolution of the two electrons after the first electron tunneled and the electron-electron interaction are described by the classical equations (in atomic unit):

$$
\frac{d^{2} \mathbf{r}_{1}}{d t^{2}}=-\frac{2 \mathbf{r}_{1}}{r_{1}^{3}}+\frac{\mathbf{r}_{1}-\mathbf{r}_{2}}{\left|\mathbf{r}_{1}-\mathbf{r}_{2}\right|^{3}}-\mathbf{F}(t),
$$




$$
\frac{d^{2} \mathbf{r}_{2}}{d t^{2}}=-\frac{2 \mathbf{r}_{2}}{r_{2}^{3}}-\frac{\mathbf{r}_{1}-\mathbf{r}_{2}}{\left|\mathbf{r}_{1}-\mathbf{r}_{2}\right|^{3}}-\mathbf{F}(t),
$$

where $\mathbf{F}(t)$ is the laser field.

In our model, the initial state of the inner electron of the Helium is described by a microcanonical distribution which is widely used in the classical-trajectory Monte Carlo (CTMC) methods established and developed by 13, 14. The CTMC method has been successfully used in studying the interaction of atoms with strong laser fields by numerous authors [15,16], which provides a statistical distribution of all the parameters defining the initial conditions of a trajectory of the electrons in the ground state of a hydrogen-like atom. Then, the initial distribution of the inner electron is

$$
\rho\left(\mathbf{r}_{2}, \mathbf{p}_{2}\right)=\frac{\delta\left(E_{2}-H_{0}\left(r_{2}, p_{2}\right)\right)}{K},
$$

where $H_{0}\left(r_{2}, p_{2}\right)=p_{2}^{2} / 2 m_{e}-Z e^{2} / r_{2} ; K$ is the normalization constant; $E_{2}=-2 a . u$. is the eigenenergy of the inner electron. Integrating the above equation, one obtains the momentum distribution

$$
\rho\left(\mathbf{p}_{2}\right)=\frac{8 p_{c}^{5}}{\pi^{2}\left(p_{2}^{2}+p_{c}^{2}\right)^{4}},
$$

in which $p_{c}^{2}=2 m_{e} U, U$ is the negative energy of the inner electron.

The spherically symmetric ground-state $\mathrm{He}^{+}$is represented by the above microcanonical distribution. This state is specified by the binding energy of the electron in the target atom and five additional parameters randomly distributed in the following ranges: $-\pi \leq \phi \leq \pi,-1 \leq \cos \theta \leq 1,-\pi \leq \eta<\pi, 0 \leq \epsilon^{2} \leq 1$ and $0 \leq \chi_{n} \leq 2 \pi$ (Ref. [14). Here, $\epsilon$ is the eccentricity of the orbit, $\chi_{n}$ is a parameter of the orbit proportional to time, and $\phi, \theta$ and $\eta$ are Euler angles. A random distribution of these parameters corresponds to equal probability of the inner electron having any phase in its periodic motion. Here, $10^{4}$ initial points are chosen and their momentum distribution is compared with Eq. (4). Figure 1 shows that they are agreeable.

The initial condition of the tunneled electron, under the SAE approximation of $\mathrm{He}^{+}$, is determined by a equation including the effective potential given in Ref. [17] and a generalized tunneling formula developed by Delone et al. [12]. In parabolic coordinates, the Schrödinger equation for a hydrogen-like atom in a uniform field $\epsilon$ is written (in atomic unit),

$$
\frac{d^{2} \phi}{d \eta^{2}}+\left(\frac{I_{p 1}}{2}+\frac{1}{2 \eta}+\frac{1}{4 \eta^{2}}+\frac{1}{4} \epsilon \eta\right) \phi=0
$$

in which $I_{p 1}=-0.9$ a.u. is the negative ionization potential of the outer electron.

The above equation has the form of the one-dimensional Schrödinger equation with the potential $U(\eta)=-1 / 4 \eta-$ $1 / 8 \eta^{2}-\epsilon \eta / 8$ and the energy $K=\frac{I_{p 1}}{4}$. The turning point, where an electron born at time $t_{0}$, is determined by $U(\eta)=K$. In the quasistatic approximation, the above field parameter $\epsilon$ relates to the laser field amplitude $F(t)$ by $\epsilon=F\left(t_{0}\right)$. One must point out, as $\epsilon>F_{t h}$, the turning point will be complex, which determines the threshold value of the field $F_{t h}=0.338$ a.u.

The evolution of the outer electron is traced by launching a set of trajectories with different initial parameters $t_{0}$ and $v_{1 x 0}$, where $v_{1 x 0}$ is the initial velocity perpendicular to the polarization of the electric field. The initial position of the electron born at time $t_{0}$ is given by $x_{10}=y_{10}=0, z_{10}=-\eta_{0} / 2$ form the Eq. (5). The initial velocity is set to be $v_{1 y 0}=v_{1 z 0}=0, v_{1 x 0}=v_{10}$. Thus, the weight of each trajectory is evaluated by [12]

$$
\begin{gathered}
w\left(t_{0}, v_{10}\right)=w(0) w(1), \\
w(1)=\frac{\sqrt{2 I_{p 1}} v_{10}}{\epsilon \pi} \exp \left(-\sqrt{2 I_{p 1}} v_{10}^{2} / \epsilon\right),
\end{gathered}
$$

and where $w(0)$ is the tunneling rate in the quasistatic approximation [18].

Before we go further, we would like to compare our model with a similar model [19] describing the double ionization of helium. First, in our model the initial condition of the inner electron is given by the classical trajectory Monte Carlo method (CTMC); Second, the Coulomb interaction is described by the real Coulomb potential. These improvements are essential. In the model given in Ref. [19, the inner electron is assumed to be rest at the center. This initial condition confines the motion of both electrons in the same plane defined by the polarization axis and the direction of the initial transverse momentum., i.e., in fact, the calculations in their paper is a $2 \mathrm{D}$ system, which may increase the 
probability of the collisions between the two electrons. On the other hand, the soften Coulomb potential approximation adopted in Ref. [19] makes the inner electrons more easily to be excited and cause an overestimation of the double ionization rate. Our model has been employed to understand the momentum distribution of the recoil ions and shown a good agreement with the experimental records [20].

In our calculation, the Eqs. (11) and (2) are solved in a time interval between $t_{0}$ and $13 T$ by employing the standard Runge-Kuta algorithm. After ten optical cycles the electric field is switched off using a $\cos ^{2}$ envelope during three cycles, and during the last two optical cycles the electrons is free from the electric field. So, the electric field can be expressed as

$$
\mathbf{F}(t)=a(t) F \cos (\omega t) \mathbf{e}_{z},
$$

where $F$ and $\omega$ are the amplitude and frequency of the field respectively and the envelope function $a(t)$ is defined by

$$
a(t)=\left\{\begin{array}{cc}
1 & t \leq 10 T \\
\cos ^{2} \frac{(t-10 T) \pi}{6 T} & 10<t \leq 13 T \\
0 & t>13 T
\end{array} .\right.
$$

The wavelength is $\lambda=780 \mathrm{~nm}$, which is so chosen to match the experiment [2], and the intensities ranging from $I=10^{14} \mathrm{~W} / \mathrm{cm}^{2}$ to the threshold value $I=4 \times 10^{15} \mathrm{~W} / \mathrm{cm}^{2}$.

In our computations, $10^{5}$ or more initial points are randomly distributed in the parameter plane $-\pi / 2<\omega t_{0}<\pi / 2$, $v_{1 x 0}>0$ for the outer electron and in the microcanonical distribution for the inner electron. The probability for double ionization and the angular distribution can be obtained by making statistics on an ensemble of classical trajectories weighed by the (6). The results have been tested for numerical convergence by increasing the number of trajectories.

In our treatment, the behavior of the classical trajectories play an important role and determine the ionization dynamics of the electrons. There are four kinds of typical trajectories. Fig. 2(a) shows a simple behavior: After tunneled out, the outer electron will be driven mainly by the field and directly run away. It collides neither with core nor with the inner electron. Fig. 2(b) gives a more complicated picture in which multiple returns and long-time trapping is experienced by the outer electron: The outer electron first tunneled out, and then oscillate in the combined laser and Coulomb fields. After several optical periods, it collides with the core and then absorb enough energy to escape. In the above two cases, no double ionization occurs since the collision between the two electrons is slight. Fig. 2(c) and 2(d) give the typical pictures of the double ionization process. In Fig. 2(c), the outer electron is born at the regime close to the peak of the electric field, then it oscillates in the combined laser and Coulomb fields. After several optical periods, it returns back to the neighborhood of the core and collides strongly with the inner electron. This collision provide enough energy for the inner bounded electron to get free. Fig. 2(d) shows that after the outer electron is tunneled, the laser field will reverse its direction within less than a quarter of the optical periods, so that this electron will be driven back when the laser field reverses its direction and collides with the inner electron near the core and make it ionized. As we will show later, the resulting energy spectra and the angular distribution of the photoelectrons for the two processes are quite distinct.

To match the experiments, Figure 3 shows the double ionization yields of helium calculated by making use of our model at 13 different intensities in the range $4 \times 10^{14}-4 \times 10^{15} \mathrm{~W} / \mathrm{cm}^{2}$. The dashed line is the single-ionization yields of He predicted by the ADK tunneling rate [1], and the solid line is the ADK tunneling rate for $\mathrm{He}^{+}$. For peak intensities below $3 \times 10^{15} \mathrm{~W} / \mathrm{cm}^{2}$, one sees that the double ionization rate obtained from our numerical simulations is larger than the ADK tunneling rate, but for the intensities above $3 \times 10^{15} \mathrm{~W} / \mathrm{cm}^{2}$, the ADK tunneling rate increases rapidly and becomes larger than the ionization rate given by our model. This figure reads that our calculation is able to reproduce, qualitatively at least, the excessive double ionization observed in helium experiments [2]. The inset in Figure 3 shows the double ionization rate calculated by our model normalized to the ADK tunneling rate of He versus the intensity. Our result is in good agreement with the data in the knee regime observed in experiments [2]: $\mathrm{He}^{2+} / \mathrm{He}^{+}$ratio in the knee regime is nearly around 0.002 . At lower intensities $\left(I<0.5 \times 10^{15} / \mathrm{cm}^{2}\right)$, the deviation between our calculation and the experimental records becomes serious. In conclusions, our model provides a suitable description for the double ionization in the knee regime, where as shown above the classical collisional trajectories (Fig.2c,d) are believed to be the main source of the double ionization. Above this regime, the tunneling ionization of the inner electron will become a dominating process and the ADK description is available. Below this regime, the ionization mechanism of the outer electron transits from tunneling regime to the multiphoton regime and the tunneling description is no longer available.

Figure 4 shows the relations between the ionization rate and the phase of the laser field when the outer electron tunneled. One finds that the most double ionization yields come from the region $\left(-0.2<\omega t_{0}<0.4\right)$ close to the peak of the electric field. There is a tail for the regime $\omega t_{0}>0.4$ and a 'cut off' for the $\omega t_{0}<-0.2$. We know that when the outer electron tunneled out near the peak of the laser field, its canonical momentum is almost zero. Hence, 
the outer electron tends to oscillate in the combined laser and Coulomb fields for several optical periods, and then return back to the neighborhood of the core to collide with the inner electron. In this case, the typical trajectory of the double ionization process corresponds to Fig. 2(c). For phase $\omega t_{0}<-0.2$, the tunneled electrons have a nonzero canonical momentum directing outwards from the core. Consequently, it will be driven by the laser field and run away directly from the core. That is, in this process, the outer electron has no chance to return to the core and no double ionization occurs in the region. For phase $\omega t_{0}>0.4$, the outer tunneled electron has a nonzero canonical momentum towards the core, and soon after it tunneled out the laser field also reverses its own direction to the same direction. So the electron will be driven back to the core by the external field and collide with the inner electron. The Fig. 2(d) shows the typical trajectory for this case. In this region the tunnel ionization of the outer electron is not efficient, and the double ionization rate is low. Comparing the two typical processes of the double ionization, one can find some intrinsic difference. In the Fig. 2(c) the outer electron was firstly ionized out, then driven by the field to collide with the inner electron and cause the double ionization, which shows a typical picture of the 'recollision' process. In the Fig.2(d) the inner electron was ionized during the process when the outer electron was driven away from core by the external field, both electrons ionize simultaneously which possesses the properties of the 'shake-off' mechanism. The difference of the two processes manifests clearly in the energy evolution of the two electrons. As shown in Fig. $5(\mathrm{a})$, the outer electron is ionized free with a positive energy, then it comes back to collide with the inner electron. This collision causes an sudden increment on the energy of the inner electron which becomes free soon. Because the collisions between the two electrons is almost instantaneous so that the energy is conserved approximately when collision happens. In the case of Fig. 5(b),during the escape process of the outer electron it collides with the inner one. Consequently, both electrons are ionized free almost simultaneously. From our calculation we know that both processes contribute to the double ionization in the knee region, but the main contribution comes form the 'recollision' process which gives more than $80 \%$ of the double ionization yields.

From our calculations, we can also obtain the photoelectron spectra (PES) and the photoelectron angular distribution (PAD). Figure 6 shows the total photoelectron energy distribution at $1 \times 10^{15} \mathrm{~W} / \mathrm{cm}^{2}$ and $1.6 \times 10^{15} \mathrm{~W} / \mathrm{cm}^{2}$ (both of them are in the knee regime) calculated from our model. On can see that, in absolute units, an increasing laser intensity results in the increase of higher energy photoelectrons. But if one scales the energy units by the pondermotive energy $U_{p}=e^{2} F^{2} / 4 m_{e} \omega^{2}$, of electron, one will find that the PES for both intensities will show similar shape: The spectrum exhibits a sharply decreasing slope (region $\mathbf{I}, 0-2 U_{p}$ ) followed an extended plateau up to $8 U_{p}$ or more (region II). This spectrum structure is much close to experimental observations in this regime [2].

We know that in our model the electron is initiated in the phase interval $[-\pi / 2, \pi / 2]$, so the total photoelectron angular distribution (PAD) has to consider the contribution of electrons originated in $[\pi / 2,3 \pi / 2]$ which is the mirror image with respect to $90^{\circ}$. Figure $7(\mathrm{a})$ is the total angular distribution of ionized electrons. One finds that except for a main concentration in the field direction, there exists a long tail decreasing with a power-law dependence $1 /\left(\sin ^{r}(\theta / 2)\right)$ which is different from the angular distribution for the pure tunneling nature where it decreases exponentially. This structure is due to the scattering with the core and the interaction between two electrons during the rescattering processions. Figure 7(b) shows the angular distribution of photoelectrons in the energy region II. The most striking feature of the plots is the existence of a slight slope up to $40^{\circ}$ followed by a tail up to $90^{\circ}$. If one compares this result with the angular distribution of the transition region in rescattering processes described in Ref. [10], where there is no photoelectrons emitted at angles much larger than $40^{\circ}$, it is not difficult to find that the tail structure is due to the electron-electron interaction. Because the velocity direction of the inner electron is random when the collision happens, the instantaneous strong interactions can give rise to large emission angle of the photoelectron. This can be verified in Figure 7(c), which shows the angular distributions of the inner electron and outer electron in double ionization respectively. This case corresponds to strong electron-electron interaction and the angular distributions is almost flat and the decreases is slowly when the emission angle increases.

In fact, the final energy and angular distribution of the photoelectrons are greatly determined by the scattering processes. The process shown in Fig. 2(a) only provides a relatively low energy for the photoelectron (region I) and gives small emission angle (the field direction). However, for the process shown in Fig 2(b), the classical trajectories have complex behavior, and the energy exchange in this process is also complicated. The multiple returns and longtime trapping can produce high energy electron in this process. So, this region contributes to the high energy part of PES. It is also found that the structures of PES and PAD are similar to some extent to the rescattering model of hydrogen 10]. This fact indicates that the main structures of PES and PAD come from the rescattering process of the outer electron with the ion $\mathrm{He}^{+}$.

In conclusions, a quasistatic two step model is used to investigate the double ionization of helium in intense linearly polarized field. Our calculations reproduce the excessive double ionization and the photoelectron spectra observed in experiments. We argue that the classical collisional trajectories are the main source of the double ionization in the knee regime and responsible for the unusual angular distribution of the photoelectrons. Two distinguished typical collisional trajectories correspond to the 'recollision' process and the 'shake-off' process respectively. Both of the two processes have contribution to the double ionization, but the 'recollision' gives the main contribution and leads 
to more than $80 \%$ of the double ionization yields. Our calculations based on the classical trajectories provide an intuitionistic picture of the double ionization of helium, and are helpful in understanding the complicated behavior of multi-electrons atoms in intense laser fields, in the way of quantum approach and the future experiments observations.

We acknowledge very helpful discussions with Dr. T.W. Cheng. This work was supported by the Important Fundamental Researching Project of China.

[1] D.N. Fittinghoff, P.R. Bolton, B. Chang, and K.C. Kulander, Phys. Rev. Lett. 69, 2642 (1992).

[2] B. Walker, B. Sheehy, L.F. DiMauro, P. Agostini, K.J. Schafer, and K.C. Kulander, Phys. Rev. Lett. 73, 1227 (1994)

[3] B. Sheehy, R. Lafon, M. Widmer, B. Walker, L.F. DiMauro, P.A. Agostini, and K.C. Kulander, Phys. Rev. A 58, 3942 (1998)

[4] M.V. Ammosov, N.B. Delone, and V.P. Krainov, Sov. Phys. JETP 64, 1191 (1986)

[5] P. Corkum, Phys. Rev. Lett. 71, 1994 (1993)

[6] K.C. Kulander, J. Cooper, and K.J. Schafer, Phys. Rev. A 51, 561 (1995)

[7] B. Walker, E. Mevel, B. Yang, P. Berger, J.P. Chambaret, A. Antonetti, L.F. DiMauro, and P.A. Agostini, Phys. Rev. A 48 R894 (1993)

[8] D.L. Fittinghoff, P.B. Bolton, B. Chang, and K.C. Kuander, Phys. Rev. A 49, 2174 (1994)

[9] K. Kondo, A. Sagiska, T. Tamida, Y. Nabekawa, and S. Watanabe, Phys. Rev. A 48, R2531 (1993)

[10] B. Hu, J. Liu and S.G. Chen, Phys. Lett. A 236, 533 (1997)

[11] J. Chen, J, Liu and S.G. Chen, Phys. Rev. A 61, 033402 (2000)

[12] N. B. Delone, and V. P. Krainov, J Opt. Soc. Am. B 8, 1207 (1991)

[13] R. Abrines and I.C. Percival, Proc. Phys. Soc. 88, 861 (1966); J.G. Leopold and I.C. Percival, J. Phys. B 12, 709 (1979)

[14] J.S. Cohen, Phys. Rev. A 26, 3008 (1982)

[15] C.H. Keitel and P.L. Knight, Phys. Rev. A 51, 1420 (1995); G. Bandarage, et al., ibid. 46, 380 (1992); M. Gajda, et al. ibid. 46, 1638 (1992); G.A. Kyrala, J. Opt. Soc. Am. B 4, 731 (1992)

[16] J. Liu, S. G. Chen and D. H. Bao, Comm. Theor. Phys. 25, 129 (1996)

[17] L.D. Landau, E. M. Lifishitz, Quantum Mechanics. (Rergamon, Oxford, 1977)

[18] A.M. Perelomov, V.S. Popov and V.M. Teren'ev, Zh. Eksp. Teor. Fiz. 50, 1393 (1966); M.V. Ammosov, N.B. Delone, and V.P. Krainov, ibid 91, 2008 (1986)

[19] T. Brakec, M.Yu. Ivanov and P. Corkum, Phys. Rev. A 54 , R2551 (1996)

[20] J. Chen, J. Liu, L.B. Fu and W.M. Zheng, Phys. Rev. A, to be published as a Rapid Communication.

\section{FIGURES CAPTION:}

Fig. 1. Momentum distribution of the initial state of the inner electron. the full circles denote the results of $10^{4}$ Monte Carlo points, the solid line is the distribution given by the formula (位).

Fig. 2. Four typical trajectories in our calculation. (a) The final energy is $E_{1}=4.694$ a.u.and $E_{2}=-2.01$ a.u., and the final angle of the outer electron $\theta=2.26$; (b) The final energy is $E_{1}=8.683 a$ a.u.and $E_{2}=-1.153 a . u$., and the final angle of the outer electron $\theta=5.67$. (c) The typical trajectories of electrons in double ionization corresponds to $\omega t_{0}$ in the phase interval $(-0.2,0.4)$. The initial conditions are $\omega t_{0}=-0.087$, the weight of the trajectory 0.168 ; the final energy is $E_{1}=3.407$ a.u., $E_{2}=3.278$ a.u., and the final angle of two electrons: $\theta_{1}=25.15, \theta_{2}=30.86$. (d) The typical trajectories of electrons in double ionization corresponds to the phase interval $\left(\omega t_{0}>0.4\right)$. Here $\omega t_{0}=0.924$, the weight of the trajectory 0.014 ; the final energy is $E_{1}=2.663$ a.u., $E_{2}=0.237$ a.u., and the final angle of two electrons: $\theta_{1}=19.2, \theta_{2}=129.0$.

Fig. 3. Numerically calculated the double ionization yields of He in our model. The dashed and dotted lines correspond to the single ionization yields of $\mathrm{He}$ and $\mathrm{He}^{+}$predicted by ADK tunneling ionization respectively; the full circles correspond to the results of our calculation. Inset: Intensity dependence of $\mathrm{He}^{2+} / \mathrm{He}^{+}$ratio given by our model. The solid line is gotten from the experiment [2].

Fig. 4. The double ionization of He versus the phase of the laser field at the moment when the outer electron tunneled.

Fig. 5. The energies evolution of the two electrons during the double ionization process. (a) corresponding to the case of Fig. 2(c), and (b) corresponding to the case of Fig. 2(d). The solid line represents the outer electron and the dashed line represents the inner electron.

Fig. 6. Photoelectron energy spectra calculated from our model. 
Fig. 7. Photoelectron angular distribution at $2 \times 10^{15} \mathrm{~W} / \mathrm{cm}^{2}$. (a) shows the total distributions of photoelectrons; (b) is the PAD for energy region II. (c) PAD of the inner electron and outer electron in double ionization. 
Distribution

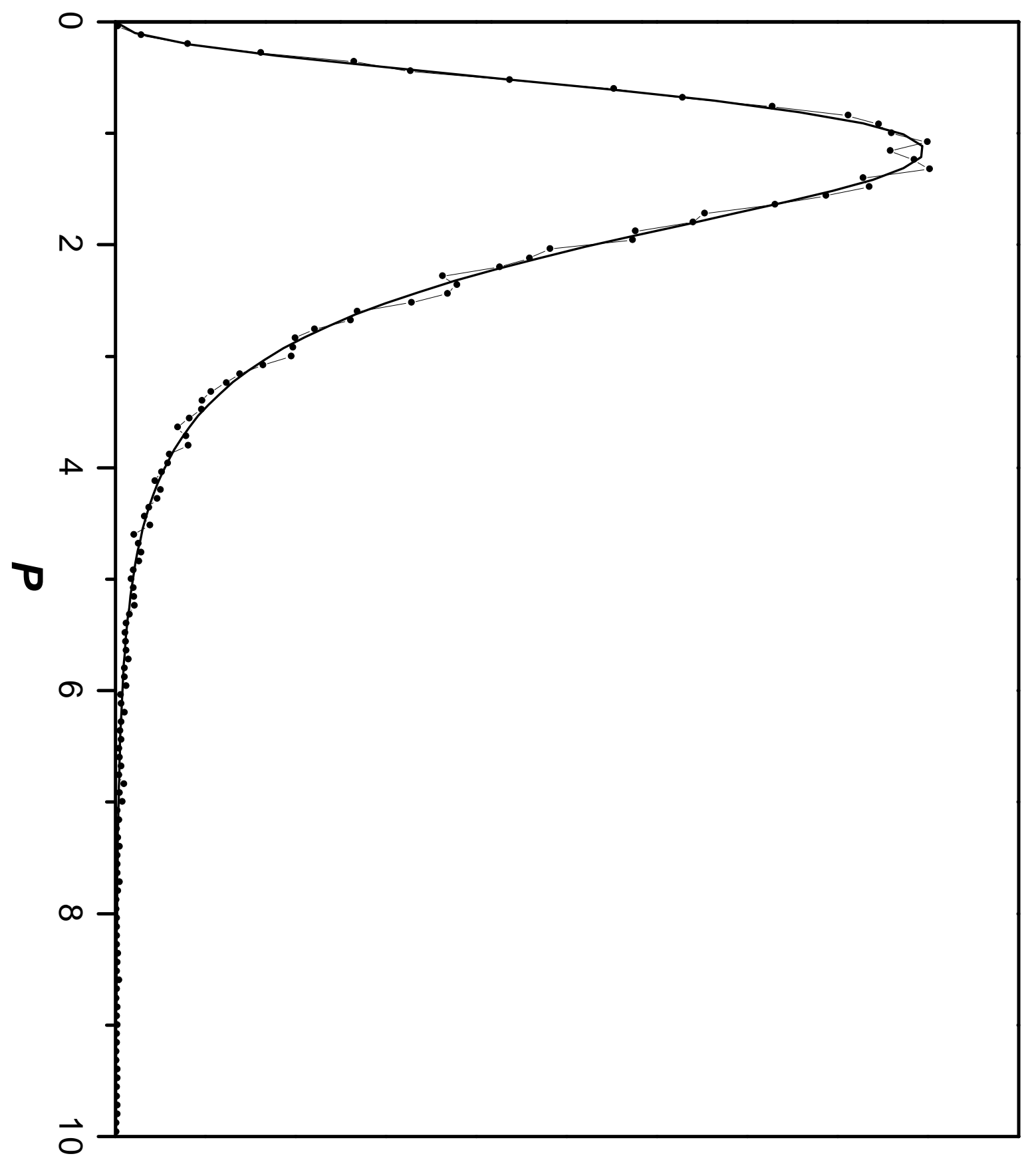




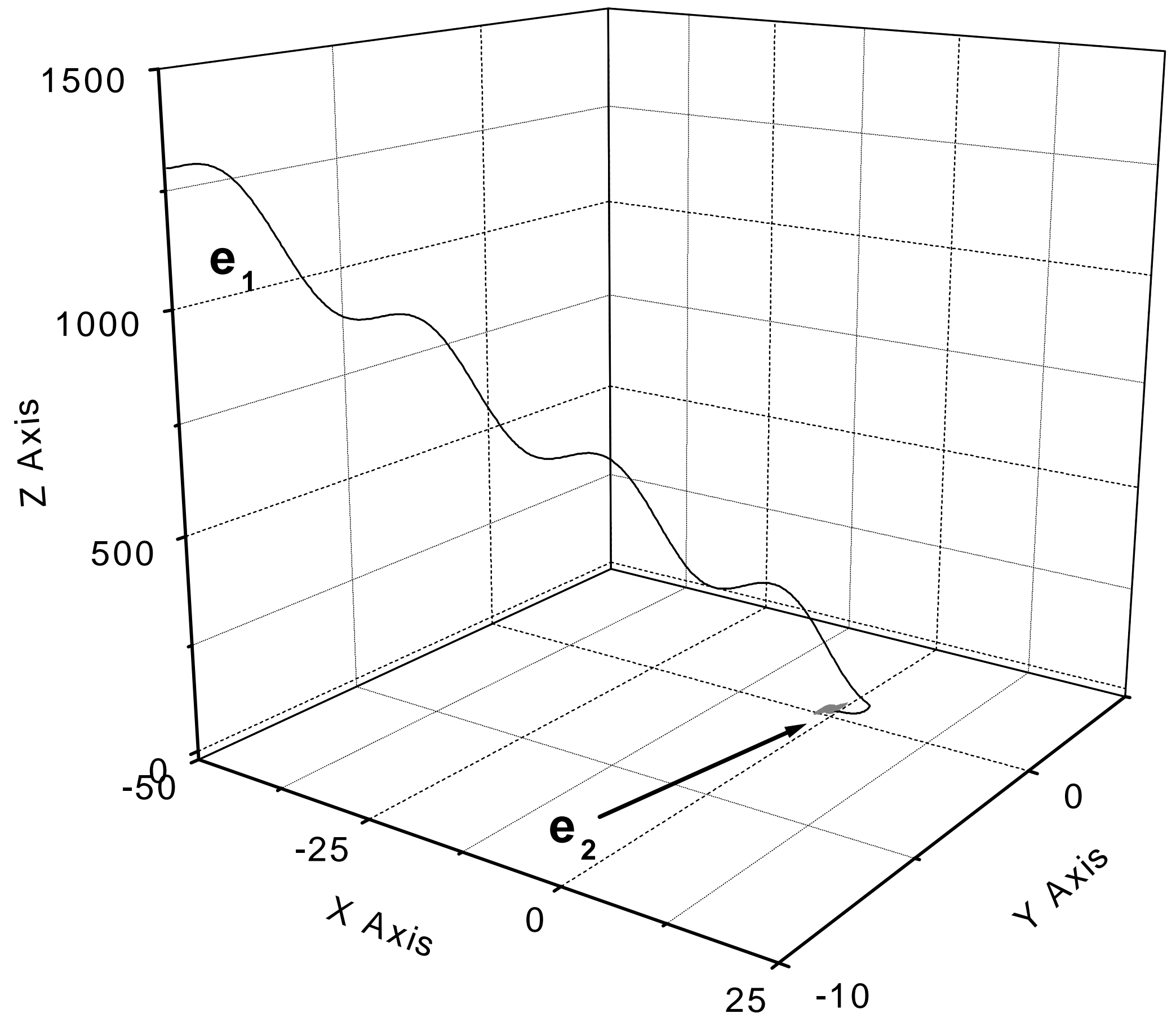




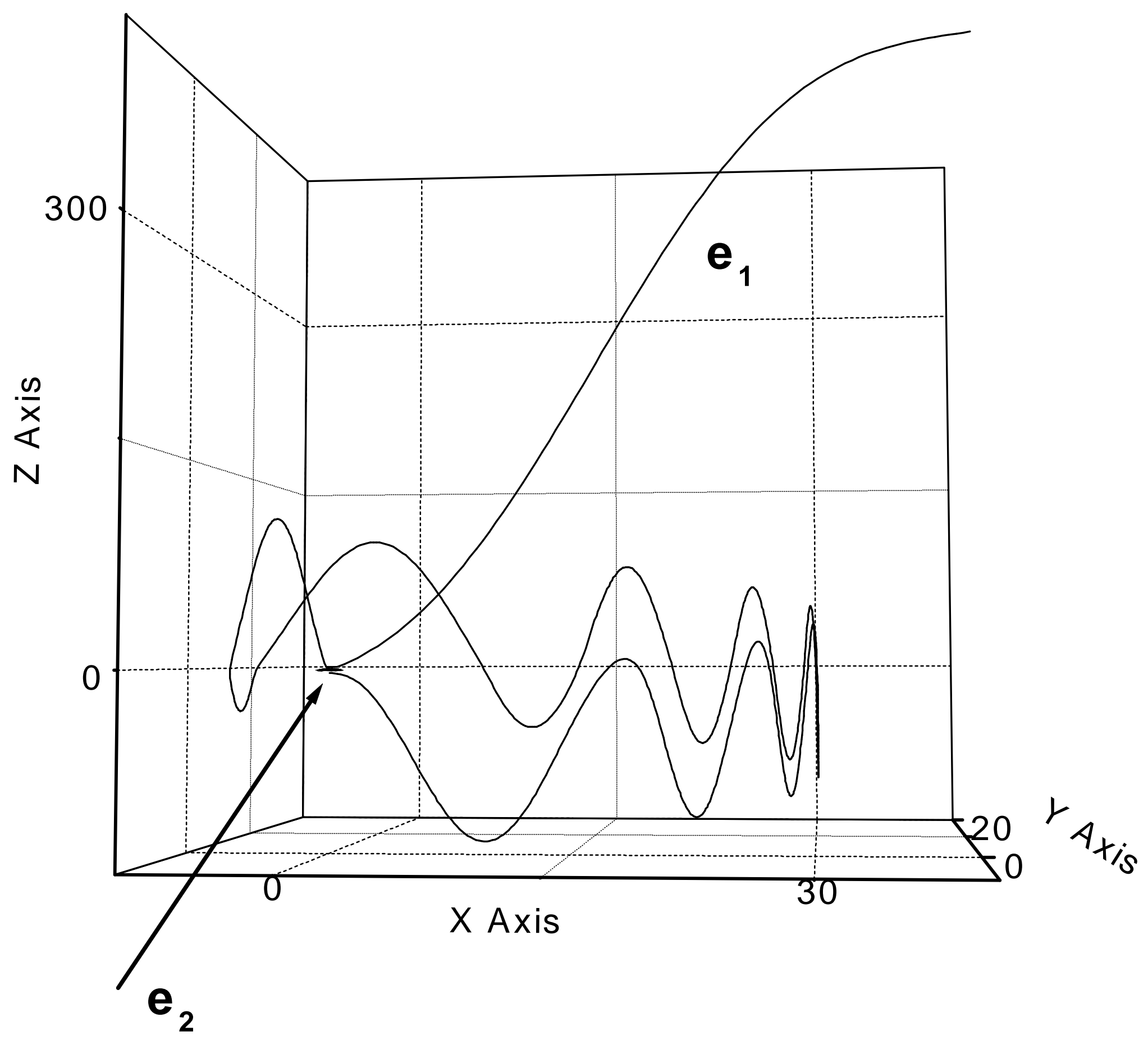




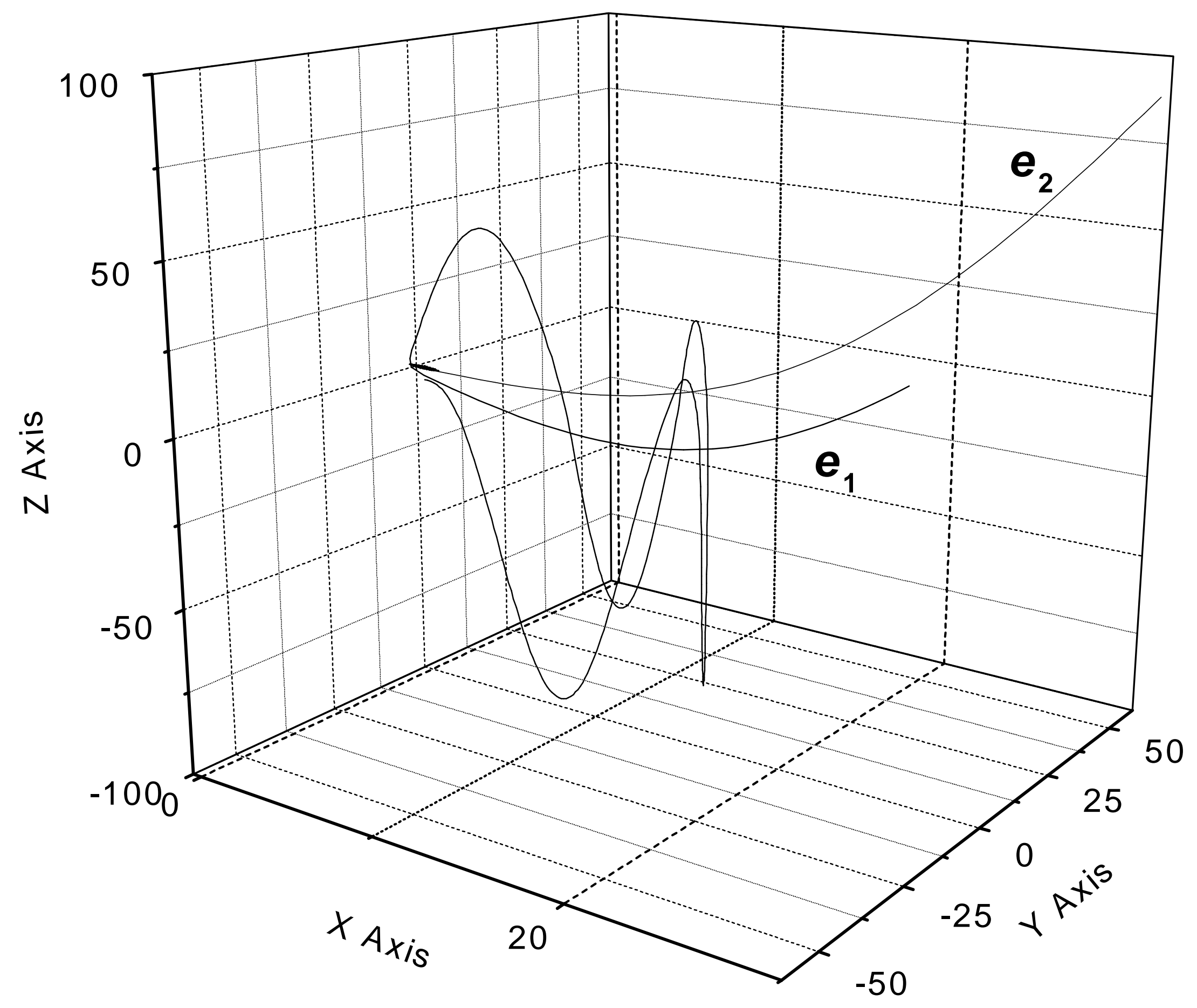




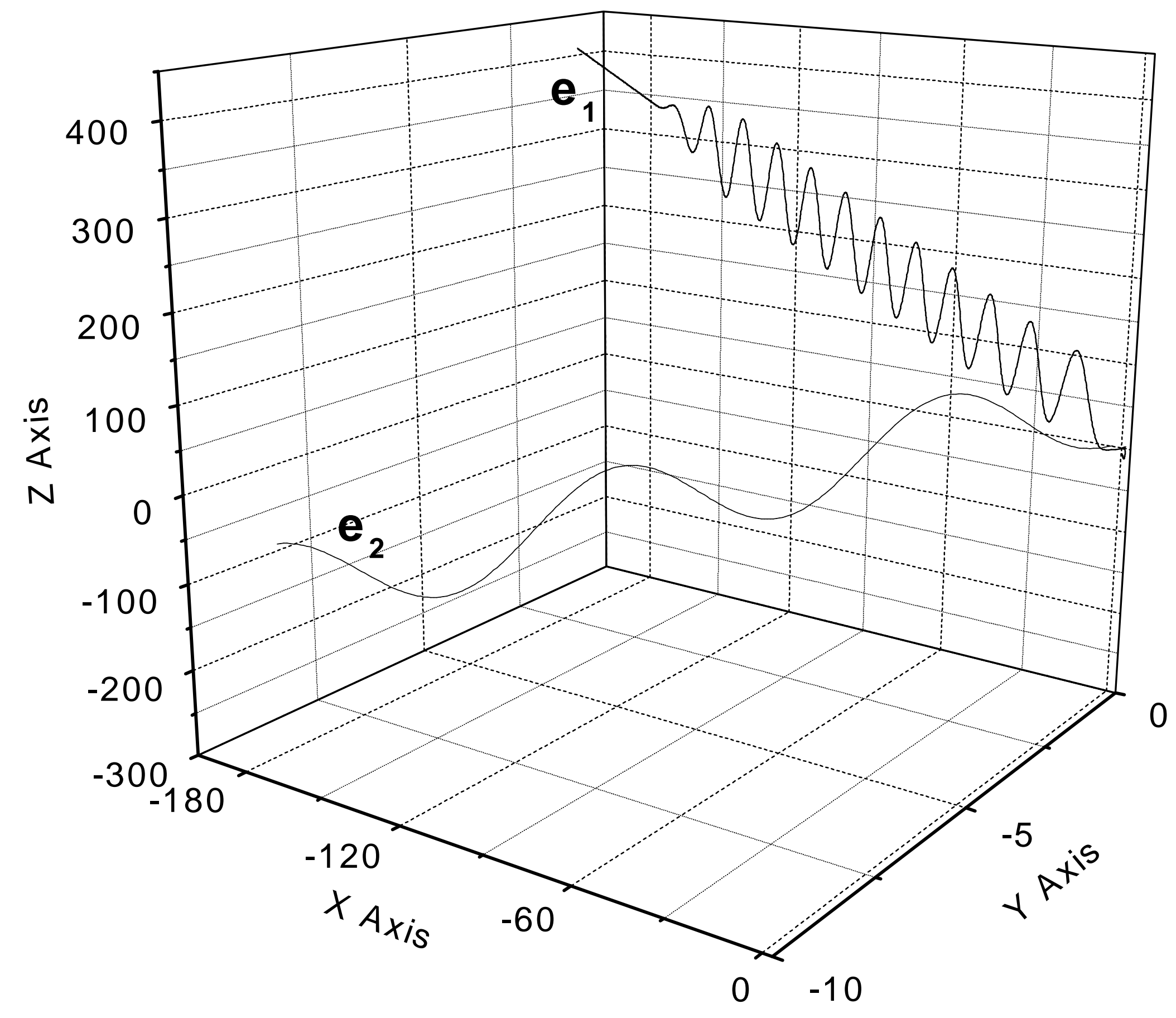




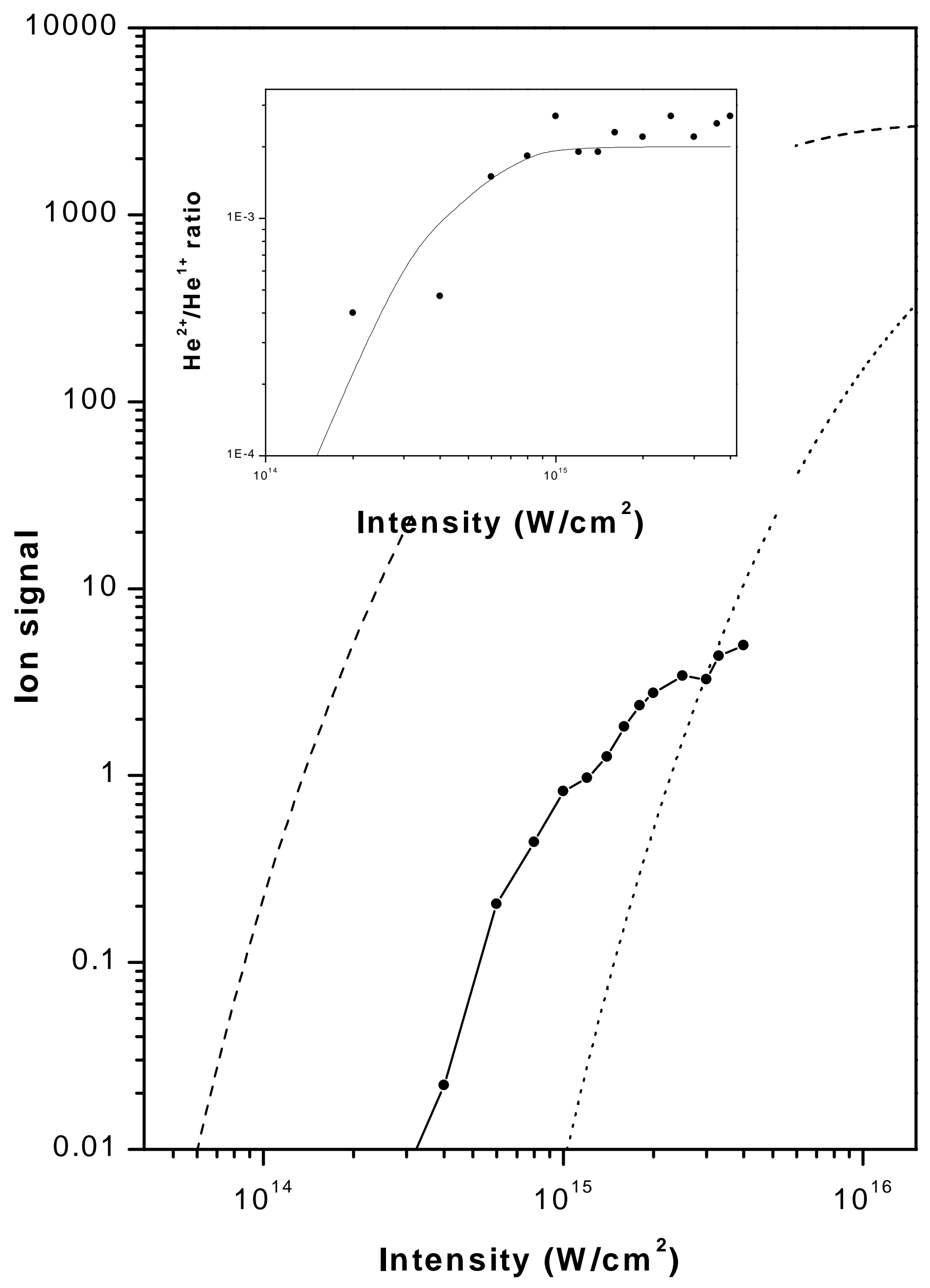




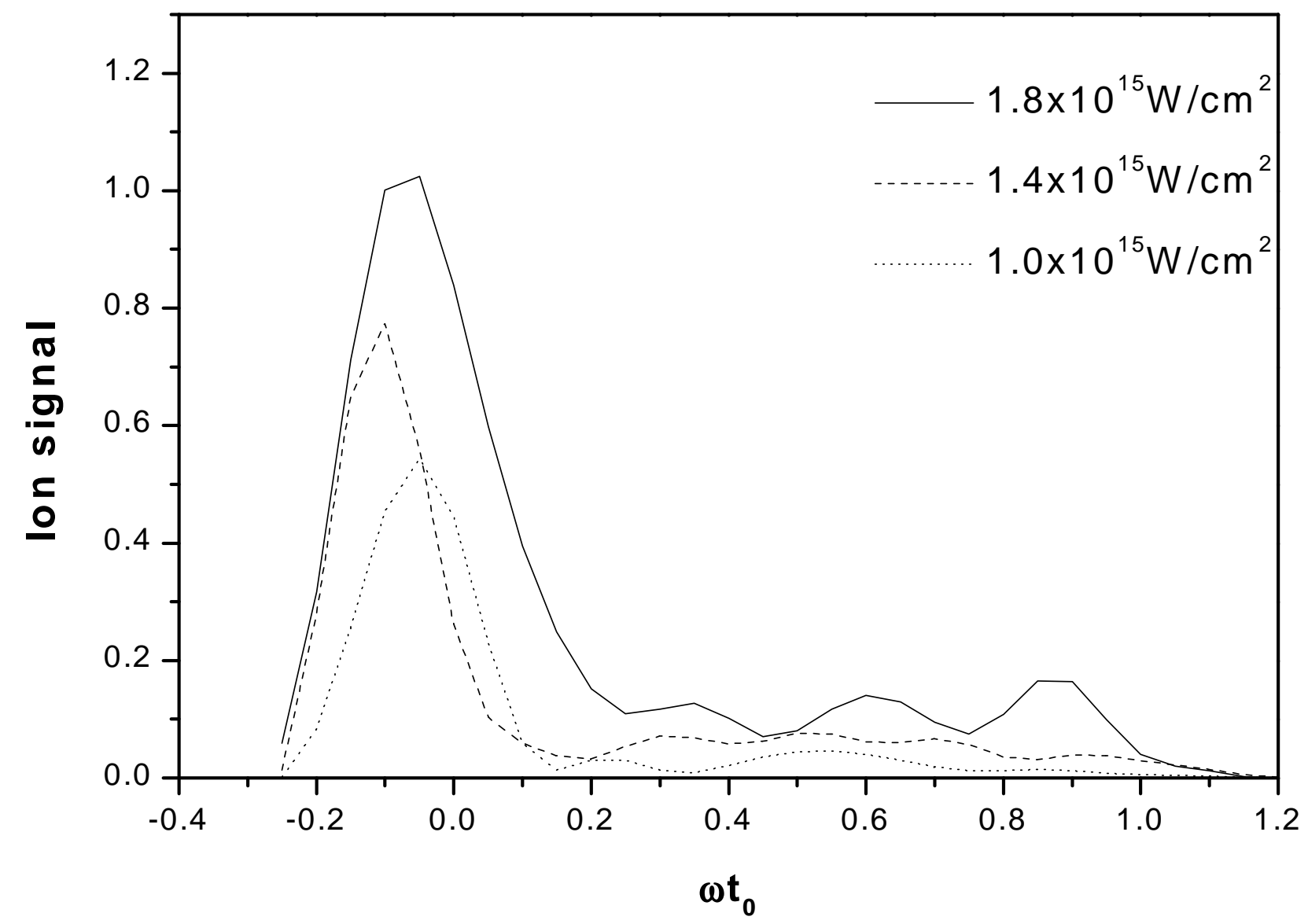



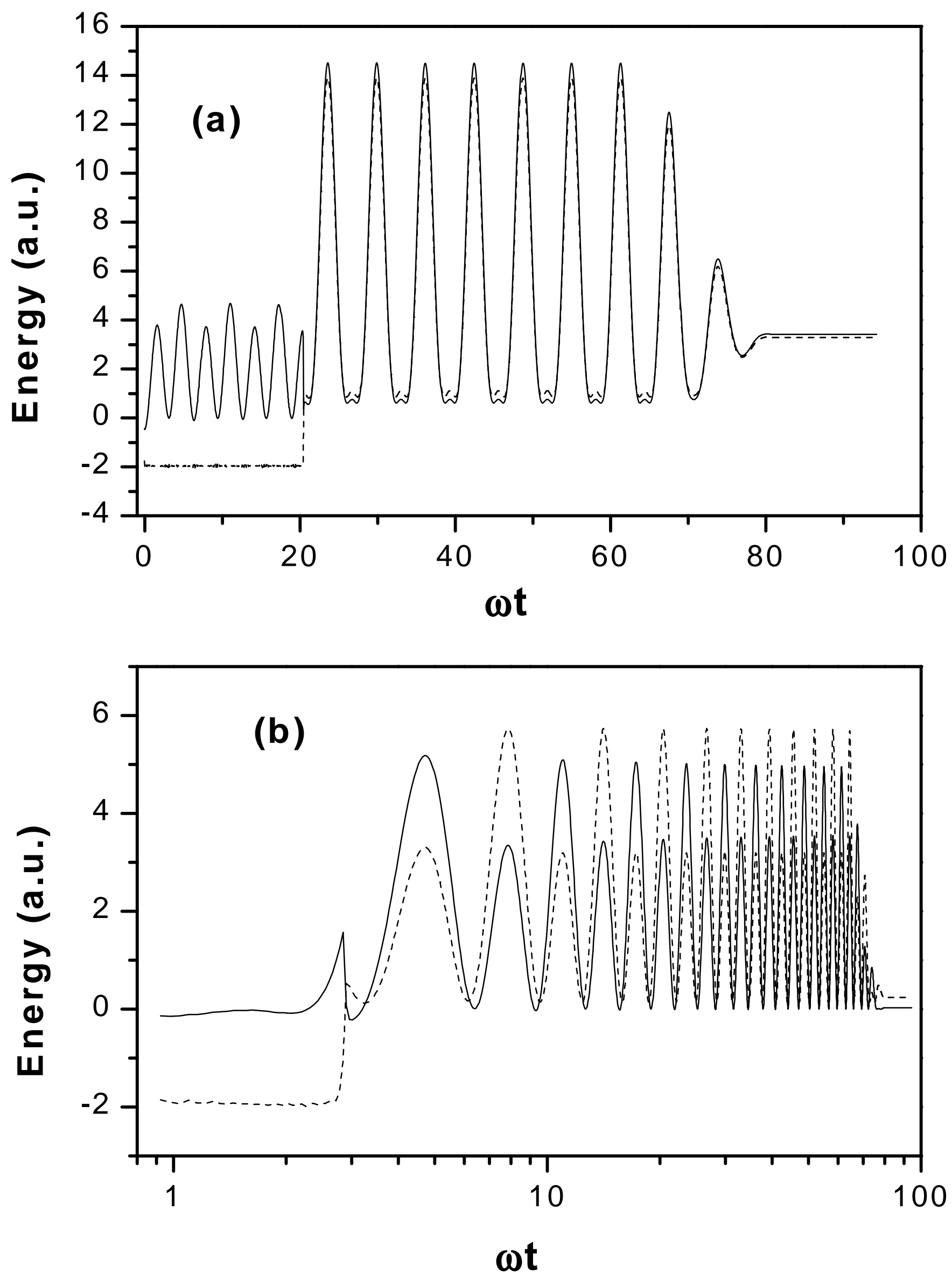


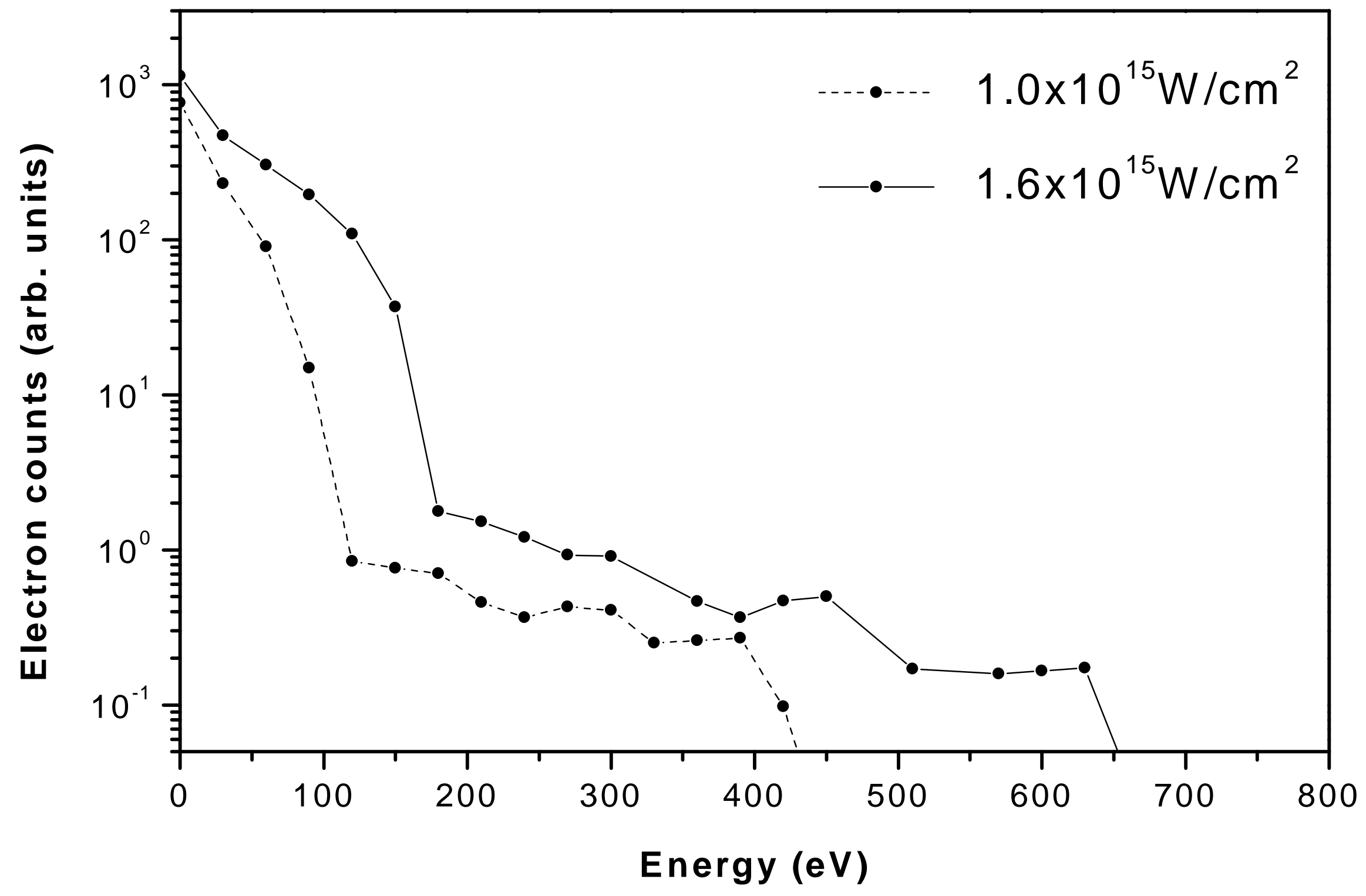




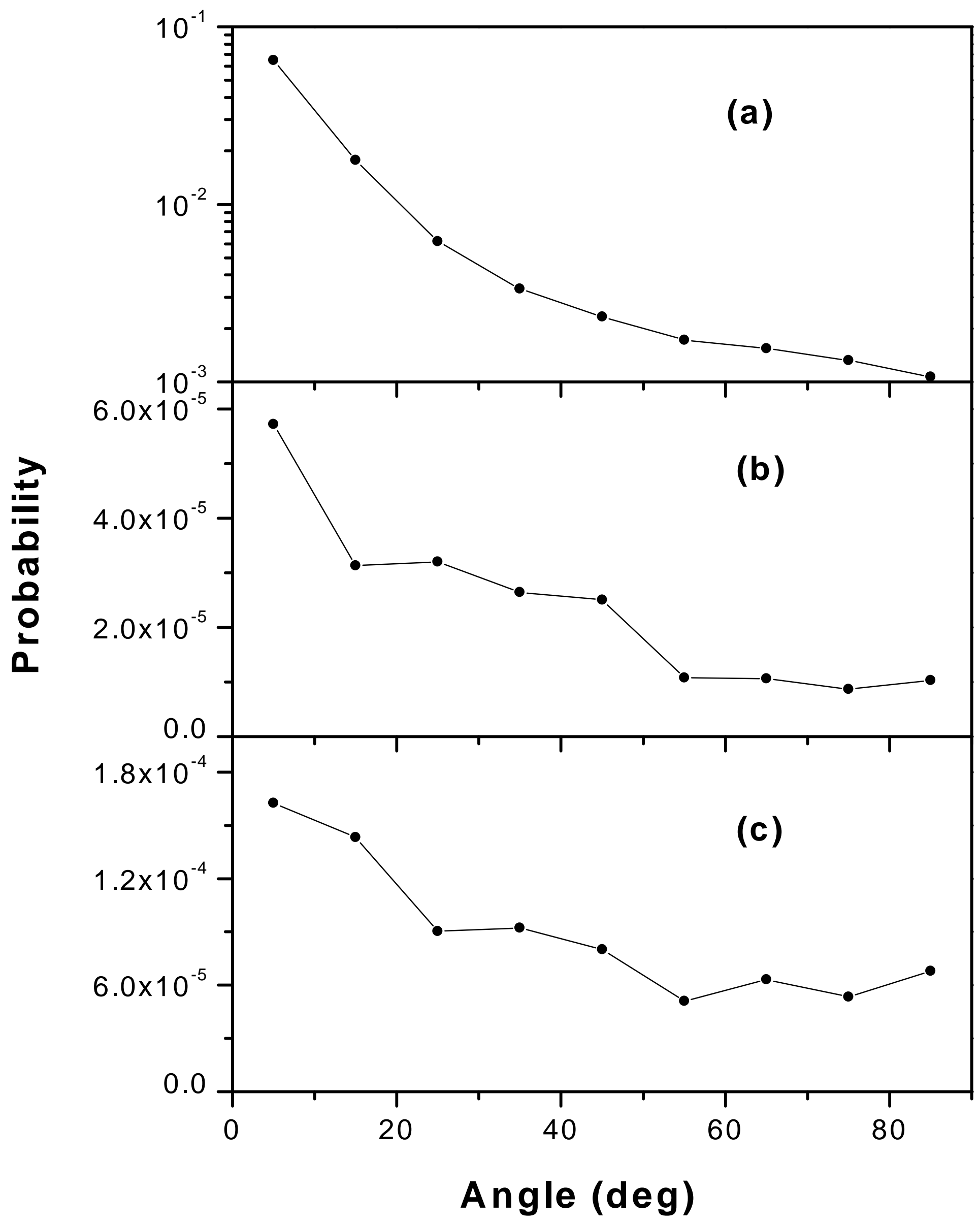

